\title{
ATUAÇÃO DO PROFISSIONAL DE EDUCAÇÃO FÍSICA NO SISTEMA ÚNICO DE SAÚDE: REVISÃO SISTEMÁTICA
}

\section{Physical educator's performance in the unified health system: a systematic review Actuación del profesional de educación física en el sistema único de salud: revisión sistemática}

\author{
Anderson dos Santos Carvalho \\ Universidade de São Paulo - EEFERP-USP - Ribeirão Preto (SP) - Brasil \\ Pedro Pugliesi Abdalla \\ Universidade de São Paulo - EEFERP-USP - Ribeirão Preto (SP) - Brasil
}

Carlos Roberto Bueno Júnior

Universidade de São Paulo - EEFERP-USP - Ribeirão Preto (SP) - Brasil

\section{RESUMO}

Objetivo: Descrever e analisar os tipos de atuação do profissional de Educação Física no Sistema Único de Saúde (SUS). Métodos: Trata-se de um estudo de revisão sistemática da literatura realizado nos bancos de dados Google Acadêmico e Scielo. Foram analisadas publicações entre os anos de 2005 e 2016 por meio dos descritores: Sistema Único de Saúde, Atividade Física e Educação Física. Resultados: Foram encontrados inicialmente 85 artigos, dos quais somente 12 se enquadraram nos critérios de inclusão e entraram nesta revisão. Poucos estudos $(n=4)$ documentam a atuação do profissional de Educação Física no SUS, a qual está voltada prioritariamente à população idosa e realizada de forma curativa. Conclusão: Considerando que o profissional de Educação Física no SUS tem sua participação restrita a apenas alguns tipos de atuação, ela necessita aumentar em abrangência e espectro de forma considerável, de modo a intervir em diferentes grupos populacionais e contribuir significativamente com a promoção da saúde.

Descritores: Saúde Pública; Atividade Motora; Atenção Primária à Saúde; Administração de Serviços de Saúde; Gestão em Saúde.

\section{ABSTRACT}

Objective: To describe and analyze types of professional practice of physical educators in the Unified Health System (Sistema Único de Saúde - SUS). Methods: Systematic literature review carried out on Google Scholar and Scielo. Publications from 2005 to 2016 were selected using the following descriptors: Unified Health System, Exercise and Physical Education. Results: A total of 85 studies were found, but only 12 met the inclusion criteria and were included in this review. Few studies $(n=4)$ address the professional practice of Physical Educators in the SUS, which is primarily targeted at older people and curative treatments. Conclusion: Considering that Physical Educators' participation in the SUS is restricted to only some types of professional practice, there is a need to increase in a considerable way its comprehensiveness and spectrum so that they can intervene in different population groups and significantly contribute to health promotion.

Descriptors: Public Health; Motor Activity; Primary Health Care; Health Services Administration; Health Management.

\section{RESUMEN}

Objetivo: Describir y analizar los tipos de actuación del profesional de Educación Física del Sistema Único de Salud (SUS). Métodos: Se trata de un estudio de revisión sistemática de la literatura realizado en los bancos de datos del Google Académico y Scielo. Fueron analizadas las publicaciones entre 2005 y 2016 a través de los descriptores: Sistema Único de Salud, Actividad Física y Educación Física. Resultados: A principio se encontraron 85 artículos de los cuales solamente 12 se encuadraron en los criterios de inclusión y entraron para la revisión. Pocos estudios $(n=4)$ documentan la actuación del profesional de la Educación Física en el SUS la cual está dirigida prioritariamente para la población mayor y realizada de manera curativa. Conclusión: Considerando que el profesional de Educación Física del SUS tiene su participación restricta a solamente algunos tipos de actuación, hay la necesidad del aumento de su utilización y espectro de manera considerable para intervenir en distintos grupos de población y contribuir de manera significativa para la promoción de la salud.

Descriptores: Salud Pública; Actividad Motora; Atención Primaria de Salud; Administración de los Servicios de Salud; Gestión en Salud. 


\section{INTRODUÇÃO}

A Política Nacional de Promoção da Saúde (PNPS) aprovou a institucionalização da promoção da saúde no Sistema Único de Saúde (SUS). Este processo apresentou conceitos e a práxis do cotidiano do SUS, como também a importância da análise da situação em saúde e suas circunstâncias no Brasil para a eleição de prioridades e focalização das ações sanitárias. Por meio dessa abordagem epidemiológica, foram eleitas áreas temáticas prioritárias para implementação da PNPS. Dentre elas, destacase a indução de atividade física/prática corporal e a importância de viver ativo como fator de proteção à saúde ${ }^{(1)}$.

A Portaria 2.608, de 28 de dezembro de 2005, apresentada pela PNPS, destina recursos aos estados da federação para investimentos em projetos locais e incentivo à prática de atividade física para diminuir os hábitos do sedentarismo, pois podem causar doenças cardiovasculares e metabólicas ${ }^{(2)}$.

Nas últimas décadas, tem-se observado uma série de mudanças no processo saúde-doença da sociedade brasileira e verifica-se hoje o aumento acelerado da morbidade e da mortalidade causadas por doenças não transmissíveis ${ }^{(1)}$. O processo de industrialização apresentou mudanças significativas no padrão de morbimortalidade mundial. Por meio desse processo, nota-se um crescimento em doenças causadas pela hipocinesia ${ }^{(2)}$. A diminuição da prática de atividade física regular é considerada fator de risco primário e independente para muitos agravos à saúde da população, particularmente para doenças cardiovasculares e metabólicas ${ }^{(3)}$. Apesar das evidências apresentadas sobre os benefícios da prática de atividades físicas regulares para a saúde e a implementação da Portaria 2.608, a literatura tem discutido e apresentado a existência de níveis populacionais de atividade física ainda ineficientes de acordo com a proposta do SUS ${ }^{(2)}$.

A prática de atividade física está relacionada com uma melhor saúde e qualidade de vida. Nos últimos anos, ficou evidenciada a importância da manutenção de um estilo de vida ativo para a saúde da população em geral ${ }^{(3,4)}$ e, apesar do crescimento de iniciativas, programas e campanhas em prol de um estilo de vida mais ativo(2) e do reconhecimento da importância da prática de atividade física como fator de promoção da saúde e de prevenção de doenças, a prevalência de exposição a baixos níveis de atividade física é elevada e parece afetar pessoas de todas as idades ${ }^{(3,4)}$.

A participação do profissional de Educação Física nas Unidades Básicas de Saúde do SUS foi regulamentada no ano de 2005, pois a atividade física e as práticas corporais foram consideradas prioridades pelo Ministério da Saúde em busca de induzir a redução do fator de risco do sedentarismo ${ }^{(1)}$. A promoção da saúde pode ser definida como o processo de capacitação dos sujeitos e da comunidade para atuar na melhoria da sua qualidade de vida e saúde, incluindo uma maior participação dos sujeitos no controle desse processo ${ }^{(1)}$, sendo considerado um dos elementos principais da atenção básica à saúde, em que o papel dos profissionais da saúde está pautado em promover ações/intervenções com objetivo de induzir mudanças nos hábitos, favorecendo melhorias nas condições de vida da população.

O objetivo do presente estudo é descrever e analisar os tipos de atuação do profissional de Educação Física no Sistema Único de Saúde (SUS).

\section{MÉTODOS}

Trata-se de um estudo de revisão sistemática da literatura que utilizou os bancos de dados Google Acadêmico e Scielo. O período de publicação dos artigos selecionados foi de 2005 a 2016, pois a atuação do profissional de Educação Física no SUS recebeu regulamentação a partir do ano 2005.

Os descritores utilizados foram: Sistema Único de Saúde, Atividade Física e Educação Física, selecionando-se: a) estudos clínicos, de caso e aleatorizados, além de estudos de revisão relacionados à atuação profissional da Educação física no SUS; b) estudos publicados no idioma português (tanto as bases de dados como o idioma foram definidos a partir do objeto de estudo). Foram excluídos os artigos com informações incompletas, ineficientes ou com irrelevância nos critérios metodológicos.

Inicialmente, os artigos foram selecionados conforme os critérios descritos acima. Após a seleção, realizaram-se análises dos títulos, resumos e textos completos. Os estudos foram organizados em quadros com o intuito de sistematização. Por fim, ocorreram as análises críticas dos conteúdos, considerando o mérito científico de cada estudo e possíveis relações de similaridade ou conflito entre eles. Esse processo foi realizado por dois pesquisadores de forma independente.

\section{RESULTADOS}

Após consulta às bases de dados Google Acadêmico e Scielo, encontraram-se 85 publicações sobre o assunto. No entanto, apenas 12 estudos foram incluídos na presente análise após a filtragem da busca, conforme critérios de seleção.

A figura 1 representa o fluxograma de seleção dos estudos. Os achados encontrados nos estudos foram divididos em dois eixos temáticos: 1- Benefícios da atividade física orientada para melhora da qualidade de vida dos usuários do Sistema Único de Saúde; 2 - Atuação do profissional de Educação Física no SUS. 


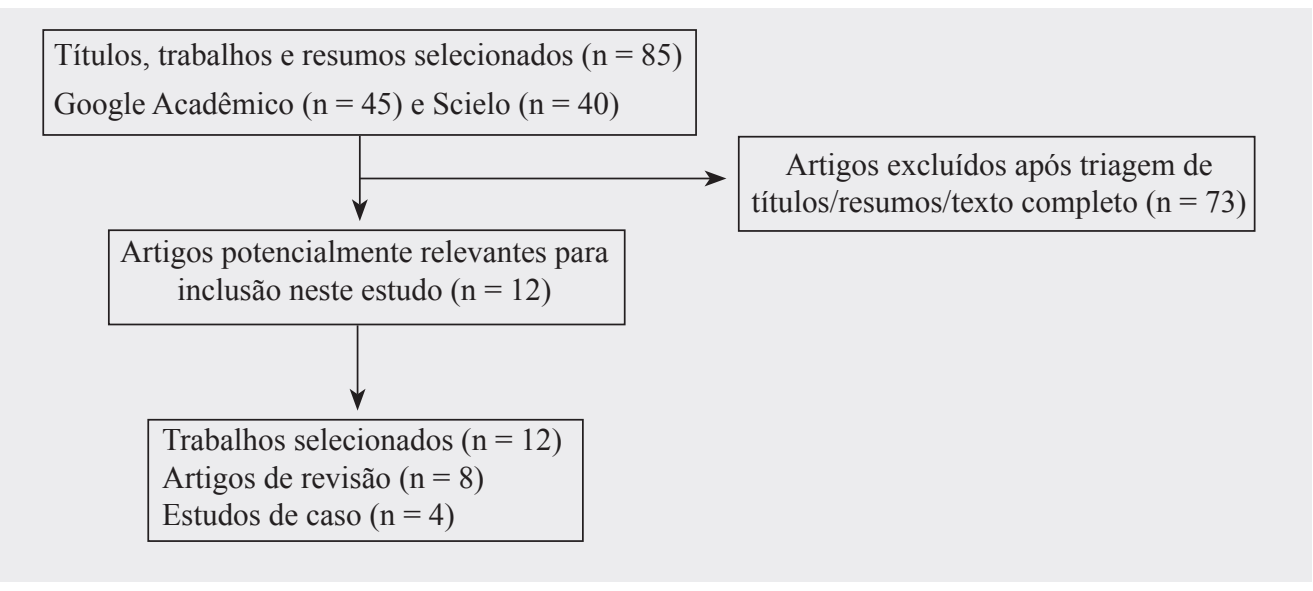

Figura 1 - Fluxograma de seleção dos artigos encontrados.

\section{DISCUSSÃO}

\section{Benefícios da atividade física orientada para melhora da qualidade de vida dos usuários do Sistema Único de Saúde}

Embora os estudos descrevam a Educação Física como parte integrante da equipe multidisciplinar na área da saúde, sua prática ainda não é bem consolidada e definida no $\operatorname{SUS}^{(5)}$, porque ainda não está bem definida a demanda específica para a atuação do profissional de Educação Física e as equipes de saúde das unidades básicas de saúde ainda ignoram o potencial de atuação do núcleo da Educação Física na prevenção, promoção, atenção ou reabilitação da saúde. Existem muitas incertezas e muito desconhecimento sobre a atuação desses profissionais. Além disso, em alguns locais onde existe demanda, a Educação Física restringe-se somente às questões que envolvem a estética, o emagrecimento ou o "combate" às doenças crônicas, como hipertensão e diabetes mellitus ${ }^{(5)}$.

As questões acima são relevantes e necessitam de atenção, porém não são únicas, uma vez que a prática da atividade física envolve benefícios que extrapolam os limites impostos pelo desconhecimento de sua potencialidade. Trata-se de uma intervenção que tem como objetivo contribuir para a qualidade de vida do paciente, possibilitando benefícios na convivência familiar, na comunidade e na prevenção ou reabilitação da saúde ${ }^{(6)}$.

A atividade física é considerada um dos principais fatores que favorecem um estilo de vida saudável, podendo atuar na prevenção ou reabilitação de muitas doenças ${ }^{(7)}$. Ela é entendida como qualquer movimento corporal produzido pelos músculos esqueléticos que resulta em dispêndio energético acima dos níveis esperados de repouso e envolve um comportamento multifacetado, em que devem ser incluídas variáveis, como escolha das atividades físicas (há inúmeras opções de exercícios, modalidades esportivas, jogos, lutas, danças e ginásticas) e ordem na qual serão realizadas; duração (tempo); frequência (número de vezes); intensidade; consumo de oxigênio e frequência cardíaca. A atividade física pode ser vista também como meio de gasto energético (GE), independentemente da atividade realizada. Diante das inúmeras variáveis de controle que devem ser monitoradas e levadas em conta durante a prescrição adequada da atividade física, ninguém melhor para prescrevê-la do que o próprio profissional de Educação Física ${ }^{(8)}$.

Por meio de uma orientação programada e direcionada de forma individual ou coletiva, os usuários podem aumentar seus níveis de atividade física, obtendo diversos benefícios físicos, mentais e sociais, como aumento da resistência, da força muscular, da capacidade cardiorrespiratória, da flexibilidade articular, do equilíbrio, da coordenação, do emagrecimento e do bem-estar psicológico, além de prevenir doenças crônicas degenerativas ${ }^{(9)}$.

\section{Atuação do profissional de Educação Física no SUS}

Apenas quatro artigos científicos relacionam de forma efetiva Educação Física, atividade física, SUS e possíveis intervenções com os usuários. Com base nos artigos científicos selecionados na presente revisão, nota-se uma carência de trabalhos que envolvam a atuação do profissional de Educação Física e suas intervenções no SUS. Serão apresentados a seguir os objetivos de cada trabalho, as intervenções e os benefícios acarretados à população envolvida.

Um estudo $^{(6)}$ teve como objetivo ampliar a discussão sobre a importância da atividade física na terceira idade por meio da Educação Física e da promoção da saúde. O projeto teve o intuito de assegurar uma melhor qualidade de vida para os idosos evolvidos. A atividade física foi oferecida no Programa Saúde da Família (PSF), por meio do projeto Ação e Saúde em Guanambi, Bahia. As atividades foram realizadas duas vezes por semana e duas vezes ao dia, tendo duas horas cada sessão. As atividades físicas realizadas foram recreação, jogos pré-esportivos, brincadeiras, massagens, alongamento, atividades de roda e exercícios aeróbicos específicos, como caminhada e ginástica com baixo impacto. Havia também algumas atividades de 
força, que consistiam em exercícios de circuito com thera band, halteres (garrafas pet) e bastões. O projeto também envolveu avaliação física e análise da composição corporal. É conhecido que atividades físicas realizadas de forma orientada podem contribuir para uma melhora na qualidade de vida dos praticantes ${ }^{(7-9)}$ e os resultados do projeto confirmam esta citação, pois houve melhoria desse aspecto e da saúde na população atendida. Houve também mudanças no estilo de vida dos sujeitos, na consciência social, na autoestima, no combate ao estresse, além de melhora no aspecto da mobilidade dos participantes.

Outro estudo $^{(9)}$ também conduzido com idosos ( $\left.\mathrm{n}=542\right)$, no município de Feira de Santana, Bahia, analisou a frequência e fatores associados à atividade física insuficiente no lazer entre idosos residentes em áreas urbanas de um município do Nordeste do Brasil. O instrumento utilizado para coleta de dados possibilitou obter informações sociodemográficas (sexo, idade, situação conjugal, escolaridade e renda), sobre doenças (obesidade, hipertensão, diabetes, cardiopatias, entre outras) e a participação e tempo destinado à atividade de lazer, relacionando-o com a prática de atividade física ${ }^{(9)}$. Sabe-se que a hipocinesia auxilia no desenvolvimento de doenças crônicas degenerativas, que são as maiores causadoras de mortes no mundo. Sendo assim, é preciso que os profissionais da saúde redobrem sua atenção para prevenção dessas doenças ${ }^{(1,7)}$ e orientem a população de forma efetiva, principalmente os idosos.

Também é importante ressaltar que a promoção da saúde é uma das estratégias de organização da gestão do SUS. A prática de atividades físicas é eficaz e possui um baixo custo financeiro, condições que permitem sua utilização como subsídio para promoção da saúde e no controle de determinantes das condições de saúde em grupos populacionais específicos ${ }^{(1)}$.

Uma pesquisa $^{(10)}$ com 29 mulheres com idade entre 48 a 79 anos, usuárias do SUS e na pós-menopausa, assistidas na Unidade da Saúde da Família (USF) dos municípios de Marília e Echaporã, São Paulo, submetidas a um programa de atividade física com duração de seis meses e frequência de duas a cinco vezes semanais, com tempo de 30 a 50 minutos por sessão, analisou a associação entre atividade física habitual e a cognição global em mulheres. O envelhecimento é entendido como processo biológico que ocorre no ser humano e ainda não pode ser revertido, estando relacionado à genética do indivíduo, à doenças crônicas degenerativas e ao estilo de vida ${ }^{(4,10-12)}$.

O envelhecimento também está conectado com o processo cognitivo, sendo esse entendido como os fatores e funções neurológicos responsáveis pelo sujeito adquirir a capacidade de tomar decisões, agindo com inteligência em seu cotidiano. É comum notar perdas cognitivas em pessoas idosas, tais como: esquecimento de fatos recentes; diminuição da atenção e da agilidade; dificuldade para calcular e perda do raciocínio; além das lentificações de atividades motoras ${ }^{(10)}$. Estima-se que $60 \%$ das mulheres na menopausa apresentam declínio na memória, o qual é decorrente de alterações fisiológicas e relacionadas com a idade ${ }^{(13)}$, sendo a inatividade física apontada como um relevante fator de risco modificável de doenças cerebrovasculares e do declínio da função cognitiva em pessoas idosas ${ }^{(10)}$. Portanto, a prática de atividade física orientada e exercícios sistematizados podem estimular a função cognitiva dos praticantes ${ }^{(4)}$. Sendo assim, esse projeto propôs para as participantes atividades de alongamento, caminhadas e ginástica localizada. Os resultados do estudo, somados aos achados da literatura, sugerem que programas de exercício físico realizados na USF podem repercutir de forma efetiva na redução do declínio da função cognitiva de mulheres em pós-menopausa ${ }^{(10)}$.

Outro estudo ${ }^{(5)}$ teve como objetivo apresentar as atividades de competência e atuação da Educação Física nas residências multiprofissionais em saúde e as dificuldades dessa experiência. O projeto foi realizado com estudantes de graduação da Universidade Federal do Rio Grande do Sul (UFRGS), por meio do programa Residência Integrada Multiprofissional Hospitalar, com ênfase na Atenção à Saúde Cardiometabólica do Adulto (RIMHAS) e na Residência Multiprofissional em Saúde da Família (RMSF), supervisionadas pela Comissão das Residências Multiprofissionais (COREMU), visto que o programa apresenta competência e atuação no SUS ${ }^{(5)}$.

O RIMHAS desenvolve atividades práticas em diferentes campos do Hospital Universitário da UFRGS, em que os alunos de Educação Física são responsáveis pelo desenvolvimento do serviço em atenção aos pacientes cardiometabólicos e se envolvem nas atividades teóricas com o foco na preparação do serviço de pronto atendimento, clínica médica e clínica cirúrgica. No segundo programa, que acontece no Centro Integrado de Diabetes e Centro de Reabilitação Física, os discentes realizam consultas laboratoriais que contemplam avaliação física, terapia e educação em saúde, com propósito de colher informações dos pacientes ${ }^{(5)}$. O programa surgiu da necessidade de se inserir o acadêmico no SUS e prepará-lo para a prática, tendo como preparação/intervenção aulas tradicionais e rodas de conversa ${ }^{(5)}$.

Analisando o programa, pode-se dizer que os tipos de atividade realizadas foram divididas em dois anos: o primeiro ano se dá por meio de "imersão" em uma equipe de Estratégia Saúde da Família (ESF) e, no segundo ano, o residente mantém sua atuação na Unidade Básica de Saúde da Família (UBSF), mas passa a realizar também os estágios de vivência em outros serviços, tais como o Centro de Atendimento Psicossocial (CAPS), os Núcleos de Apoio à Saúde da Família (NASF), a Gestão e Vigilância Sanitária ${ }^{(5)}$. O estudo apresentou que as experiências que articulam a formação acadêmica do núcleo da Educação Física e o SUS contribuem para edificação de um caminho de diálogo entre os sujeitos atuantes nas residências multiprofissionais ${ }^{(5)}$. $\mathrm{O}$ programa apresentado vai ao encontro da PNPS no SUS, pois está preocupado com a situação da saúde dos indivíduos e amplia o conceito de saúde aos profissionais da saúde, destacando a indução de atividades físicas/práticas corporais. No entanto, é necessário aprimorar a formação do profissional de Educação Física nesse contexto ${ }^{(1)}$. 
As informações dos quatro estudos de caso apresentados nessa seção encontram-se de maneira resumida no Quadro I.

Dos quatro artigos de caso selecionados, três atenderam pessoas idosas ou acima de 48 anos, e todos com o intuito de auxiliar na melhora de alguma condição patológica já existente. O último estudo discutido foi o único que apresentou uma preocupação com a formação e preparação dos profissionais que atuam na prática da Educação Física, e teve o objetivo de complementar a formação dos profissionais para atuarem de forma efetiva no SUS. Verifica-se também nesta revisão que não foi encontrado um programa no SUS que trabalhe a questão da promoção da saúde com a população infantil, sendo também preciso que os profissionais de saúde atuem com intuito de prevenir doenças e criar hábitos saudáveis nessa fase da vida ${ }^{(14)}$.

Verifica-se que os professores/pesquisadores de Educação Física têm se aproximado cada vez mais do campo do conhecimento da saúde coletiva ${ }^{(15)}$ e tido o intuito de apresentar outros tipos e programas de atuação do profissional de Educação Física (Quadro I).

Quadro I - A atuação do profissional de Educação Física no Sistema Único de Saúde (SUS)

\begin{tabular}{|c|c|c|c|}
\hline Autor/ano & Objetivo & Método & Principais resultados/achados \\
\hline Corrêa et al. $2014^{(5)}$ & $\begin{array}{l}\text { Apresentar as atividades } \\
\text { de competência e } \\
\text { atuação da Educação } \\
\text { Física nas residências } \\
\text { multiprofissionais r e } \\
\text { as dificuldades dessa } \\
\text { experiência. }\end{array}$ & $\begin{array}{l}\text { Síntese dos dados de } \\
\text { profissionais de Educação } \\
\text { Física engajados em pós- } \\
\text { graduação lato-sensu. }\end{array}$ & $\begin{array}{l}\text { As residências multiprofissionais são } \\
\text { ambientes que permitem atuação do } \\
\text { profissional de Educação Física tanto } \\
\text { na atenção primária quanto na atenção } \\
\text { secundária à saúde. }\end{array}$ \\
\hline $\begin{array}{l}\text { Rodrigues, Costa e } \\
\text { Barros, } 2010^{(6)}\end{array}$ & $\begin{array}{l}\text { Ampliar a discussão sobre } \\
\text { a importância da atividade } \\
\text { física na terceira idade por } \\
\text { meio da Educação Física } \\
\text { e da promoção da saúde, } \\
\text { pontuando as possíveis } \\
\text { contribuições. }\end{array}$ & $\begin{array}{l}\text { Oferecimentodeduashorasde } \\
\text { atividade física ( } 2 \text { x/semana) } \\
\text { para idosos no Programa } \\
\text { Saúde da Família (PSF). As } \\
\text { atividades realizadas foram } \\
\text { recreação, jogos, massagens, } \\
\text { alongamento, atividades de } \\
\text { roda, caminhada, ginástica, } \\
\text { exercícios de circuito com } \\
\text { thera band, halteres e bastões. } \\
\text { O projeto também envolveu } \\
\text { avaliação física e análise da } \\
\text { composição corporal. }\end{array}$ & $\begin{array}{l}\text { Melhoria das condições de saúde, } \\
\text { mudanças no estilo de vida, maior } \\
\text { consciência social, autoestima, menor } \\
\text { propensão a doenças, combate ao estresse } \\
\text { e melhor mobilidade, culminando no } \\
\text { tratamento não farmacológico de doenças } \\
\text { crônicas dos idosos. }\end{array}$ \\
\hline Rocha et al. $2013^{(9)}$ & $\begin{array}{l}\text { Analisar os fatores } \\
\text { associados à atividade } \\
\text { física insuficiente no lazer } \\
\text { entre idosos. }\end{array}$ & $\begin{array}{l}562 \text { idosos responderam } \\
\text { a um formulário } \\
\text { contendo informações } \\
\text { sociodemográficas, doenças } \\
\text { referidas e participação em } \\
\text { atividades físicas no lazer } \\
\text { (autopercepção do tipo e da } \\
\text { intensidade da atividade). }\end{array}$ & $\begin{array}{l}\text { Somente } 18,3 \% \text { foram classificados } \\
\text { como ativos no lazer. Houve uma elevada } \\
\text { frequência de indivíduos inativos no } \\
\text { lazer, principalmente os de baixa renda } \\
\text { e os mais idosos. Existe a necessidade } \\
\text { de criação de políticas públicas de saúde } \\
\text { vinculadas ao SUS com espaços para } \\
\text { prática do lazer ativo por idosos. }\end{array}$ \\
\hline $\begin{array}{l}\text { Ramos e Chagas, } \\
2015^{(10)}\end{array}$ & $\begin{array}{l}\text { Analisar a associação } \\
\text { entre atividade física } \\
\text { habitual e a cognição } \\
\text { global em mulheres na } \\
\text { pós-menopausa. }\end{array}$ & $\begin{array}{l}29 \text { mulheres participantes } \\
\text { de programas de exercício } \\
\text { em Unidades de Saúde da } \\
\text { Família responderam ao } \\
\text { questionário internacional } \\
\text { de atividade física (IPAQ) } \\
\text { versão curta e ao mini exame } \\
\text { do estado mental (MEEM). }\end{array}$ & $\begin{array}{l}\text { Um maior volume de atividade física } \\
\text { com intensidade moderada ou vigorosa } \\
\text { pode contribuir para um melhor } \\
\text { desempenho da cognição de mulheres na } \\
\text { pós-menopausa. }\end{array}$ \\
\hline
\end{tabular}




\begin{tabular}{|c|c|c|c|}
\hline $\begin{array}{l}\text { Freitas, Carvalho e } \\
\text { Mendes, } 2013^{(14)}\end{array}$ & $\begin{array}{l}\text { Relacionar o conceito } \\
\text { teórico/metodológico da } \\
\text { Clínica Ampliada com } \\
\text { aspectos da formação } \\
\text { profissional em Educação } \\
\text { Física e o trabalho em } \\
\text { saúde. }\end{array}$ & Reflexivo & $\begin{array}{l}\text { Existe uma escassez na formação } \\
\text { profissional quanto à intervenção, } \\
\text { sendo predominante a perspectiva } \\
\text { biologicista. A Clínica Ampliada pode } \\
\text { ser uma alternativa, uma vez que propicia } \\
\text { comunicações com outras profissões, } \\
\text { objetivando ações orientadas com } \\
\text { relações horizontalizadas, aproximando } \\
\text { o profissional do serviço de saúde e } \\
\text { concomitantemente dos usuários do SUS. }\end{array}$ \\
\hline $\begin{array}{l}\text { Martinez e } \\
\text { Bacheladenski, } \\
2009^{(15)}\end{array}$ & $\begin{array}{l}\text { Refletir sobre a inserção } \\
\text { de professores de } \\
\text { Educação Física nos } \\
\text { Núcleos de Apoio à Saúde } \\
\text { da Família (NASF) e no } \\
\text { Programa de Residência } \\
\text { Multiprofissional em } \\
\text { Saúde da Família } \\
\text { (PRMSF). }\end{array}$ & Reflexivo & $\begin{array}{l}\text { O Programa de Residência } \\
\text { Multiprofissional em Saúde da Família } \\
\text { pode ser uma alternativa para formação } \\
\text { político-educativa e crítica de professores } \\
\text { de Educação Física para atuação no } \\
\text { SUS, uma vez que a formação atual } \\
\text { prioriza a atuação voltada para o trabalho } \\
\text { individual e o espaço privado e, por sua } \\
\text { vez, a atuação no NASF demanda o } \\
\text { entendimento do processo saúde-doença- } \\
\text { cuidado de comunidades. }\end{array}$ \\
\hline $\begin{array}{l}\text { Mendes e Carvalho, } \\
2015^{(16)}\end{array}$ & $\begin{array}{l}\text { Pensar e experimentar } \\
\text { as práticas corporais } \\
\text { em articulação com os } \\
\text { usuários, os profissionais } \\
\text { de saúde e o serviço. }\end{array}$ & Qualitativo & $\begin{array}{l}\text { O trabalho com as práticas corporais } \\
\text { apoiado pelos profissionais de saúde } \\
\text { contribuiu para ampliar a visão dos } \\
\text { usuários sobre o trabalho com o } \\
\text { corpo, tendo como meta a produção da } \\
\text { autonomia e o cuidado. }\end{array}$ \\
\hline Guarda et al. $2014^{(17)}$ & $\begin{array}{l}\text { Analisar as potenciais } \\
\text { contribuições dos } \\
\text { profissionais de Educação } \\
\text { Física ao SUS com base } \\
\text { em aspectos políticos e } \\
\text { legislação. }\end{array}$ & $\begin{array}{l}\text { Análise da legislação } \\
\text { brasileira do campo da saúde } \\
\text { (Constituição Federal), por } \\
\text { meio das leis, portarias } \\
\text { ministeriais e resoluções dos } \\
\text { conselhos nacionais de Saúde } \\
\text { e Educação. }\end{array}$ & $\begin{array}{l}\text { Desde a criação do SUS, observa-se a } \\
\text { incorporação gradativa dos profissionais } \\
\text { de Educação Física. Esses podem } \\
\text { contribuir para a elaboração de ações } \\
\text { de incentivo à prática de atividades } \\
\text { físicas, auxílio a estratégias de promoção } \\
\text { da saúde, prevenção e controle de } \\
\text { doenças, bem como compor equipes } \\
\text { multiprofissionais e contribuir no } \\
\text { enfretamento ao sedentarismo e melhoria } \\
\text { da condição de vida da sociedade. }\end{array}$ \\
\hline
\end{tabular}




\begin{tabular}{|c|c|c|c|}
\hline $\begin{array}{l}\text { Mendes et al. } \\
2014^{(18)}\end{array}$ & $\begin{array}{l}\text { Identificar e analisar as } \\
\text { atividades realizadas } \\
\text { pelos profissionais de } \\
\text { Educação Física nos } \\
\text { serviços da rede pública } \\
\text { de saúde. }\end{array}$ & $\begin{array}{l}\text { Técnica de grupo focal } \\
\text { com a participação de onze } \\
\text { profissionais. }\end{array}$ & $\begin{array}{l}\text { Os profissionais ingressam nos serviços } \\
\text { de saúde com conhecimento insuficiente } \\
\text { da área de atuação, uma vez que não } \\
\text { utilizam os conhecimentos oferecidos } \\
\text { na graduação destinados para o trabalho. } \\
\text { Os profissionais julgam ser necessária } \\
\text { a vivência do trabalho ou formação em } \\
\text { pós-graduação para correta atuação no } \\
\text { SUS. Modelos de ensino alternativo } \\
\text { ao hegemônico biológico devem ser } \\
\text { propostos, e devem incluir reflexão crítica } \\
\text { da realidade e uma nova concepção de } \\
\text { saúde, sociedade e sujeito. }\end{array}$ \\
\hline $\begin{array}{l}\text { Fraga, Carvalho e } \\
\text { Gomes, 2012 }\end{array}$ & $\begin{array}{l}\text { Acompanhar e analisar os } \\
\text { processos de composição } \\
\text { e articulação entre ensino, } \\
\text { serviço e comunidade } \\
\text { com vistas a constituir } \\
\text { uma rede de saberes e } \\
\text { práticas que responda aos } \\
\text { desafios da formação em } \\
\text { saúde comprometida com } \\
\text { a defesa e consolidação do } \\
\text { Sistema Único de Saúde. }\end{array}$ & Qualitativo & $\begin{array}{l}\text { Existe uma tímida inserção de estudantes } \\
\text { e profissionais de Educação Física } \\
\text { no SUS, comparada às das profissões } \\
\text { "irmãs" da área da saúde. Já se observa } \\
\text { interesse de pesquisadores e grupos } \\
\text { de estudo e pesquisa, isoladamente, } \\
\text { em participar dos debates no campo da } \\
\text { saúde coletiva com intuito de qualificar } \\
\text { a inserção nesses espaços da formação } \\
\text { fundamentada na saúde coletiva e na } \\
\text { intervenção orientada pelos princípios do } \\
\text { SUS. }\end{array}$ \\
\hline Santos et al. $2011^{(20)}$ & $\begin{array}{l}\text { Salientar a importância } \\
\text { do Programa de Educação } \\
\text { pelo Trabalho para a } \\
\text { Saúde (PET-Saúde) como } \\
\text { formação complementar } \\
\text { do profissional de } \\
\text { Educação Física para o } \\
\text { SUS. }\end{array}$ & Diário de campo & $\begin{array}{l}\text { A matriz curricular da maioria dos } \\
\text { cursos de graduação em Educação } \\
\text { Física é deficitária quando se trata de } \\
\text { Saúde Pública e Saúde Coletiva. O } \\
\text { PET-Saúde é uma maneira de estimular } \\
\text { e estender a formação para atuação no } \\
\text { SUS, favorecendo a interdisciplinaridade } \\
\text { juntamente com o princípio da } \\
\text { integralidade. }\end{array}$ \\
\hline $\begin{array}{l}\text { Becker, Gonçalves e } \\
\text { Reis, }(2016)^{(21)}\end{array}$ & $\begin{array}{l}\text { Sintetizar as evidências } \\
\text { disponíveis na literatura } \\
\text { referentes aos programas } \\
\text { de promoção de atividade } \\
\text { física no SUS. }\end{array}$ & Revisão de literatura & $\begin{array}{l}\text { Programas comunitários } \\
\text { aconselhamentos são as principais ações } \\
\text { para a promoção da atividade física no } \\
\text { SUS, principalmente na população adulta } \\
(\geq 18 \text { anos). }\end{array}$ \\
\hline
\end{tabular}

Dois estudos ${ }^{(14,16)}$ apresentaram o programa "Clínica Ampliada", que é uma forma de intervenção centrada no sujeito concreto, na sua existência concreta, considerando a doença como parte dessa existência. O programa, que propõe de forma efetiva os aspectos do cuidar da doença e, ao mesmo tempo, desenvolver a promoção da saúde, pode ser desenvolvido em clínicas de saúde (privadas), unidades básicas de saúde e no SUS. Um estudo ${ }^{(17)}$ apresenta três programas: o Núcleo de Apoio à Saúde da Família (NASF), formado por uma equipe de profissionais de diferentes áreas de conhecimento que atuam em conjunto com os profissionais das equipes de Saúde da Família( ${ }^{(14,15,17,18)}$; o Programa Academia da Saúde (PAS), que são pólos que oferecem aos pacientes a atenção à saúde, pautando-se sempre na perspectiva da promoção da saúde com os aspectos do cuidar, de forma que suas atividades são desenvolvidas em espaços físicos dotados de equipamentos, contam com auxílio de profissionais qualificados para contribuir com a promoção da saúde e busca oferecer modos de vida saudáveis para a população ${ }^{(17)}$; e, por fim, 
o programa Estratégia Saúde da Família (ESF), que tem como meta desenvolver diretrizes e fundamentos da atenção básica à saúde, buscando ampliar a resolutividade e impacto na situação da saúde das pessoas ${ }^{(17,20)}$.

Outro estudo $^{(18)}$ apresentou o programa de Centros de Atenção Psicossocial (CAPS), que é multiprofissional (composto por médicos, enfermeiros, psicólogos, assistentes sociais, terapias ocupacionais, profissionais de Educação Física, entre outros) e oferece atendimento diário para pessoas com transtornos mentais graves. Foi apresentado também o programa Academias da Saúde e Residências Multiprofissionais em Saúde, que atuam na área de saúde mental, atenção básica e saúde do idoso. A Academia da Saúde tem como perspectiva a promoção da saúde de seus participantes por meio da gestão do cuidado e da proposição de atividades físicas aos seus usuários. É importante salientar que as atividades são propostas por profissionais de Educação Física ${ }^{(18)}$.

Um quarto estudo ${ }^{(20)}$ apresentou três outros programas/conceitos: a) o Programa de Educação pelo Trabalho para a Saúde (PET), uma iniciativa do governo federal que busca aproximar as universidades (estudantes e docentes) dos serviço de saúde (profissionais da saúde) e dos usuários a fim de problematizar o ensino em saúde, como também qualificar a atenção e o cuidado em saúde; b) a Atenção Primária à Saúde (APS), que tem como objetivo reorganizar a assistência à saúde, valorizando as práticas de promoção de saúde e prevenção de doenças ou agravos de maneira geral em conjunto com o SUS; e c) o Programa Saúde da Família (PSF), que hoje é chamado de Estratégia Saúde da Família.

Em relação aos tipos de atuação discutidos nos artigos, alguns estudos ${ }^{(16-18,20,22)}$ apresentaram dois tipos de atuação do profissional de Educação Física: primeiro a "aula tradicional”, voltada para atividades práticas, e, segundo, a "roda de conversa", que reconhece os espaços coletivos como lugar de reflexão crítica, produção de subjetividade e constituição de sujeitos. Por fim, a técnica de grupo focal, apresentada por um estudo ${ }^{(18)}$, é direcionada para um pequeno número de sujeitos, fazendo com que o trabalho fique mais direcionado e ágil para as discussões. Essa técnica tem como objetivo identificar e possibilitar a tomada de consciência sobre um determinado tema, utilizando a tendência humana de formar opiniões e mudar de atitude na interação com o outro. Por fim, um estudo sugere que os programas comunitários e de aconselhamento são as principais ações que visam promover a atividade física no SUS para a população adulta ${ }^{(21)}$.

Os profissionais da saúde vinculados a esses programas citados acima geralmente atuam em unidades básicas de saúde, praças, bosques, escolas e universidades, entre outros espaços. Pode-se notar uma formação diversificada de profissionais nesses programas, como enfermeiro, profissional de Educação Física, fisioterapeuta, médico, nutricionista, psicólogo e terapeuta ocupacional. Os programas geralmente são mantidos pelo governo federal, estadual ou municipal e, em alguns casos, são desenvolvidos por meio de projeto de extensão universitária. Os programas mantidos pelos órgãos públicos geralmente selecionam os profissionais por meio de concursos públicos, concursos seletivos (temporários) ou por indicação política.

Existem vários outros tipos de ação que os profissionais de Educação Física poderiam efetuar no SUS, além dos já citados anteriormente, como: realizar territorialização; criar e distribuir material de divulgação com datas, horários e descrição das atividades realizadas na comunidade voltadas à saúde e à qualidade de vida; fazer visitas domiciliares; discutir problemas da comunidade por meio da participação nas reuniões dos conselhos locais e municipais de saúde; modificar os aspectos ambientais para favorecer a prática de atividade física, buscando melhores condições de segurança e iluminação, aumento das áreas verdes e resolução de questões estéticas da região, como a presença de lixo em espaços públicos e a qualidade das calçadas; reciclar materiais para as atividades práticas; promover eventos em escolas, praças, parques e unidades de saúde; além de incentivar a comunidade a participar de eventos organizados por outras instituições. Todas essas ações deveriam ser desenvolvidas por todos os profissionais de Educação Física que atuam no contexto do SUS, pois são ações primárias que ajudariam a melhorar o atendimento aos usuários ${ }^{(22,23)}$.

É necessário que o profissional de Educação Física procure, juntamente com os outros membros das equipes de saúde, conhecer o local onde atuará. Assim, é preciso levar em consideração a necessidade local por meio da caracterização da situação epidemiológica. Nesse contexto, a territorialização ${ }^{(22)}$ pode ser entendia como um auxílio de avaliação do Profissional da Saúde, pois visa a compreensão atual do processo saúde e doença da população local, apontando as variáveis biológicas, psíquicas e sociais que remetem à necessidade de ações que possam compreender e intervir nos problemas que estão afetando o território(22).

Por meio da territorialização, o profissional de Educação Física pode realizar visitas domiciliares, marcar reuniões com a comunidade e discutir os problemas atuais dos indivíduos de forma individual ou em grupo. Por meio desses encontros, além de buscar a compreensão da saúde e doença da população, é possível realizar discussões pertinentes, como conscientização da população sobre a preservação do meio ambiente (parques, bosques e praças) e a relevância desses espaços para as práticas de atividades físicas e esportivas ${ }^{(21)}$. Por fim, por meio da territorialização, o profissional de Educação Física terá acesso a elementos essenciais para discutir com outros profissionais e com a comunidade local.

Há um estudo ${ }^{(23)}$ que classifica o serviço da saúde em dois grandes grupos: 1) a prestação de assistência direta à saúde das pessoas (em consultórios, centros de saúde e hospitais); e 2) as ações sobre o meio ambiente, a fim de controlar os fatores que exercem efeitos prejudiciais à saúde. Buscar uma melhor qualidade de vida é o desafio das pessoas que abandonam a vida sedentária. Assim, se a atividade prática for realizada junto à natureza ou ao ar livre, como em parques, bosques e praças, esses ambientes contribuem para a motivação desses novos praticantes de atividade física. Para isso, é necessário que o local da prática apresente estética agradável e seja adequado para utilização da população. Toda essa organização pode ser realizada 
tanto pelos profissionais de Educação Física como em conjunto com a comunidade, gerando um trabalho de preservação e conscientização ${ }^{(22,23)}$.

Outro estudo ${ }^{(24)}$ apresenta os benefícios da preservação e conscientização dos indivíduos quando se trabalha no meio ambiente. Em uma aula realizada no bosque (passeio na trilha), os autores observaram a motivação, a cooperação, a alegria e a socialização entre alunos e professores. Assim, entende-se que a prática de atividade física realizada em conjunto com a natureza dá oportunidade para a comunidade cuidar da saúde (objetivo principal) e, ao mesmo tempo, auxilia a promoção de um trabalho educativo, propiciando um momento com a natureza e a conscientização entre professores/alunos nas questões de melhorias na segurança do local, preservação do espaço, limpeza e qualidade das calçadas e bancos. Por meio dessas atitudes, alunos e professores buscam melhorias nas condições de saúde em conjunto com os aspectos naturais ${ }^{(23,24)}$. O profissional de Educação Física pode desenvolver esse tipo de trabalho também nas escolas, um local fundamental para se trabalhar a conscientização.

Existem na literatura diversos temas que podem ser desenvolvidos pelo profissional de Educação Física, os quais podem, por exemplo, articular os indivíduos com o meio ambiente, com temas como meio ambiente, temperatura e aulas de Educação Física; Educação Física, lazer e meio ambiente; Saúde e meio ambiente; Esportes de aventura e meio ambiente. Além disso, alguns autores ${ }^{(25,26)}$ argumentam que é de extrema importância aproximar a Educação Física, o meio ambiente e a escola, uma vez que são temáticas que isoladamente vêm merecendo um destaque significativo no meio acadêmico porque vivem em constante processo de atualização e transformação. O profissional de Educação Física pode desenvolver nas escolas, por exemplo, trabalhos com materiais recicláveis e apresentar aos alunos e à comunidade a importância de reciclar e de dar utilidade aos materiais que são muitas vezes descartados de forma irregular no meio ambiente ${ }^{(27)}$ em atividades físicas, pois é possível, inclusive, dar um novo destino para os materiais por meio de sua adaptação como recursos didáticos.

O SUS foi criado e colocado em prática no final de década de 1980, tendo como princípios a universalidade, a integralidade e a equidade ${ }^{(14,19)}$. Algumas políticas têm possibilitado aos profissionais de Educação Física se institucionalizarem como participantes nos serviços de atenção à saúde básica, sendo alguns exemplos a Política Nacional de Promoção da Saúde (PNPS), a Política Nacional de Práticas Integrativas e Complementares (PNPIC), os Núcleos de Apoio de Saúde à Família (NASF), o Programa Academia da Saúde ${ }^{(14)}$, além das Residências Multiprofissionais em Saúde (áreas de Saúde Mental, Atenção Básica e Saúde do Idoso $)^{(18)}$. Apesar do crescimento de oportunidades para atuação dos profissionais de Educação Física no SUS, essa participação ainda está em sua fase inicial ${ }^{(17)}$, e fatores como a ausência do conteúdo de saúde coletiva nos cursos de graduação em Educação Física podem contribuir para a manutenção desse estágio ${ }^{(28)}$.

Além de aumentar a participação do profissional de Educação Física no SUS, é preciso criar estratégias mais efetivas para alterar o comportamento sedentário da população nesse contexto da prática profissional ${ }^{(17)}$. Propõem-se estratégias que visam melhorar as intervenções a partir de métodos diferenciados, as quais enfatizam o processo e os sujeitos em detrimento do que é tradicionalmente proposto, que tem como enfoque a "doença", tornando bases da atuação valores como afeto, acolhimento e outros teores subjetivos ${ }^{(14)}$. Porém, poucos estudos experimentaram essas diferentes abordagens metodológicas na proposição de atividades em Educação Física no SUS. Outra estratégia pertinente seria voltar a atenção ao ambiente escolar, incentivando a mudança e possivelmente a manutenção de um futuro mais ativo, tendo como princípio um caráter preventivo ${ }^{(17)}$, diferente do que foi documentado e realizado no SUS. Nesse contexto, intervenções em escolas dos profissionais de Educação Física vinculados ao SUS têm grande potencial para resultar em benefícios à saúde pública.

De acordo com os registros e trabalhos documentados sobre a atuação do profissional de Educação Física no SUS, notase que a atuação está prioritariamente voltada a remediar os efeitos de uma vida sedentária. A maior parte das intervenções trata de pessoas da terceira idade e leva em conta intervenções com caráter curativo. A potencialidade da atividade física como fator preventivo de doenças crônicas não transmissíveis e como promotora de maior qualidade de vida deve ser melhor explorada. Não foram encontrados estudos com crianças, e é a partir dessa idade que se deve trabalhar com a prevenção e a promoção da saúde. Por fim, apesar da crescente atuação dos profissionais de Educação Física no contexto do SUS, parece que esses profissionais ainda terão muito trabalho para reinventar estratégias de atuação e integrar novos saberes ao seu caráter tecnicista/biológico, aproximando-se das Ciências Humanas e da Saúde Pública, fomentando sentidos e significados às práticas de atividades físicas nas comunidades brasileiras.

\section{CONCLUSÃO}

Por meio desta revisão sistemática, é possível concluir que o profissional de Educação Física tem sua participação no SUS limitada a apenas alguns tipos de atuação. Nesse sentido, tal participação necessita aumentar de forma considerável em abrangência e espectro, de modo a intervir em diferentes grupos populacionais e contribuir de forma significativa com a promoção da saúde.

\section{REFERÊNCIAS}

1. Malta DC, Castro AM, Gosch CS, Cruz DKA, Bressan A, Nogueira JD, et al. A Política Nacional de Promoção da Saúde e a agenda da atividade física no contexto do SUS. Epidemiol Serv Saúde 2009;18(1):79-86. 
2. Hallal PC, Tenório MCM, Tassitano RM, Reis SR, Carvalho YM, Cruz KA, et al. Avaliação do programa de promoção da atividade física Academia da Cidade de Recife, Pernambuco, Brasil: percepções de usuários e não-usuários. Cad Saúde Pública. 2010;26(1):70-8.

3. Mendonça BCA, Toscano JJO, Oliveira ACC. Do diagnóstico à ação: experiências em promoção da atividade física programa Academia da Cidade Aracaju: promovendo saúde por meio da atividade física. Rev Bras Ativ Fís Saúde. 2009;14(3):211-6.

4. Groppo HS, Nascimento CMC, Stella F, Gobbi S, Oliani MM. Efeitos de um programa de atividade física sobre os sintomas depressivos e a qualidade de vida de idosos com demência de Alzheimer. Rev Bras Educ Fís Esporte. 2012;26(4):543-51.

5. Corrêa LQ, Valério MP, Teixeira AO, Guerreiro LF, Silveira DF, Machado PT, et al. A atuação da educação física nas residências multiprofissionais em saúde. Rev Bras Promoção Saúde. 2014;27(4):428-33.

6. Rodrigues ZM, Costa DS, Barros JB. Educação Física e Promoção da Saúde: Contribuição para o SUS. In: IV Congresso Centro-Oeste de Ciências do Esporte e I Congresso Distrital de Ciências do Esporte; 2010 Set 22-25; Brasília; 2015.

7. Guedes DP, Almeida FN, Tolentino JM Neto, Maia MFM, Tolentino TM. Baixo peso corporal/magreza, sobrepeso e obesidade de crianças e adolescentes de uma região brasileira de baixo desenvolvimento econômico. Rev Paul Pediatr. 2013;31(4):437-43.

8. Cafruni CB, Valadão RCD, Mello ED. Como Avaliar a Atividade Física? Rev Bras Ciênc Saúde. 2012;10(33):61-71.

9. Rocha SV, Almeida MMG, Araújo TM, Santos LB, Rodrigues WKM. Fatores associados à atividade física insuficiente no lazer entre idosos. Rev Bras Med Esporte. 2013;19(3):191-5.

10. Ramos LHN, Chagas EFB. Associação entre atividade física habitual e cognição global em mulheres pós-menopausa usuárias do SUS. Rev Bras Ciênc Mov. 2015;23(2):14-21.

11. Barros KD, Oliveira AAB, Oliveira A Filho. A influência do treinamento com pesos em mulheres acima de 50 anos. Acta Sci Health Sci. 2011;33(1):43-50.

12. Ueno DT, Gobbi S, Teixeira CVL, Sebastião E, Prado AKG, Costa JRL, et al. Efeitos de três modalidades de atividade física na capacidade funcional de idosos. Rev Bras Educ Fís Esporte. 2012;26(2):273-81.

13. Rossato LC, Contreira, Corazza ST. Análise do tempo de reação e do estado cognitivo em idosas praticantes de atividades físicas. Fisioter Pesqui. 2011;18(1):54-9.

14. Freitas FF, Carvalho YM, Mendes VM. Educação física e saúde: aproximações com a clínica ampliada. Rev Bras Ciênc Esporte. 2013;35(3):639-56.

15. Martinez JFN, Bacheladenski M. Educação física e saúde coletiva: possibilidades de inserção e formação profissional no/ para o SUS. In: XVI Congresso Brasileiro de Ciências do Esporte e III Congresso Internacional de Ciências do Esporte; 2009 Set 20-25; Salvador; 2015.

16. Mendes VM, Carvalho YM. Sem começo e sem fim... com as práticas corporais e a Clínica Ampliada. Interface Comun Saúde Educ. 2015;19(54):603-13.

17. Guarda FB, Silva RN, Araújo JL Júnior, Santana PR. Incorporação e contribuições dos profissionais de educação física ao Sistema Único de Saúde do Brasil. Tem Act Saúde Colet. 2014;8(3):185-96.

18. Mendes MFM, Moraes M, Andrade SC, Rocha CMF. Educação Física e a rede de saúde pública: dilemas, possibilidades e desafios entre a formação e a intervenção. Motrivivência. 2014;26(43):133-49.

19. Fraga AB, Carvalho YM, Gomes IM. Políticas de formação em educação física e saúde coletiva. Trab Educ Saúde. 2012;10(3):367-86.

20. Santos ALB, Maia JP Neto, Souza FCV, Oliveira BN, Rui EM, Sá MEG. Educação Física e o PET-Saúde: Uma Estratégia Complementar na Formação para o SUS. Sanare. 2011;10(2):75-8.

21. Becker LA, Gonçalves PB, Reis RS. Programas da promoção da atividade física no Sistema Único de Saúde brasileiro: revisão sistemática. Rev Bras Ativ Fís Saúde. 2016;21(2):110-22.

22. Mafra MRP, Chaves MMN. O Processo de territorialização e atenção à saúde no Programa Saúde da Família. Fam Saúde Desenv. 2004;6(2):127-33.

23. Toscano JJO. Academia de ginástica: um serviço de saúde latente. Rev Bras Ciênc Mov. 2001;9(1):40-2. 
24. Bento LCM, Ribeiro RD. Educação física e meio ambiente: nas trilhas do ecoturismo-um estudo de caso do município de Indianópolis, MG. Em Extensão. 2010;9(1):183-94.

25. Rodrigues LH, Darido SC. Educação física escolar e meio ambiente: reflexões e aplicações pedagógicas. Lect Educ Fís Deportes. 2006;11(100):1-1.

26. Oliveira WF, Alvim MPB. Educação física e educação ambiental: como trabalhar no âmbito escolar? Movimentum. 2009;4(2):1-17.

27. Rodrigue BSB, Santos TM, Silva GG, Gonçalves JB, Souza RP, Maciel RG. A importância da utilização de materiais recicláveis como material didático nas oficinas do jogo no ensino fundamental. In: $8^{\circ}$ Fórum de Ensino, Pesquisa, Extensão e Gestão; 2014 Set 24-27; Montes Claros; 2015.

28. Anjos TC, Duarte ACGO. A educação física e a estratégia de saúde da família: formação e atuação profissional. Physis (Rio de J). 2009;19(4):1127-44.

\section{Apoio: $\mathrm{CNPq}$}

\section{Endereço do primeiro autor}

Anderson dos Santos Carvalho

Universidade de São Paulo - USP

Av. Bandeirantes, 3900

Bairro: Monte Alegre

CEP: 14040-907 - Ribeirão Preto - SP - Brasil

Email: anderson.carvalho@usp.br

\section{Endereço para correspondência:}

Carlos Roberto Bueno Júnior

Universidade de São Paulo - USP

Av. Bandeirantes, 3900

Bairro: Monte Alegre

CEP: 14040-907 - Ribeirão Preto - SP - Brasil

E-mail: buenojr@usp.br 